\title{
The Impact of Vehicular Networks on Urban Networks
}

\author{
Mahkame Megan Khoshyaran \\ Economics Traffic Clinic (ETC), Paris, France \\ Email: megan.khoshyaran@wanadoo.fr
}

Received 7 August 2014; revised 5 September 2014; accepted 1 October 2014

Copyright (C) 2014 by author and Scientific Research Publishing Inc.

This work is licensed under the Creative Commons Attribution International License (CC BY). http://creativecommons.org/licenses/by/4.0/

c) (i) Open Access

\begin{abstract}
The objective of this paper is to study the impact of a vehicular network on a physical (road) network consisting of several intersections controlled by traffic lights. The vehicular network is considered to be a random graph superimposed on a regular Hamiltonian graph. The two graphs are connected by hyperlinks. The evolution of traffic at intersections given the existence of vehicular networks is measured by the method of reflective triangles.
\end{abstract}

\section{Keywords}

Road Network, Hamiltonian Graph, Vehicular Network, Hyperlinks, Queue Lengths, Delays, Reflexion Triangles

\section{Introduction}

The objective of this paper is to study the impact of a vehicular network on a physical (road) network consisting of several intersections controlled by traffic lights. The communication network is represented by what is called a Vehicular Network (VN) that is considered to be a random graph [1] [2], superimposed on a regular Hamiltonian graph (road network). The two graphs are connected by hyperlinks. Each hyperlink signifies the interaction between the random graph (vehicular network) and the Hamiltonian graph (road network). Interaction is defined as a driver being at an intersection and talking to someone on a cell phone or connecting to Internet while idly waiting for the green light. Each hyperlink is characterized by $(0,1),(0)$ signifying waiting at an intersection without using a cell phone and (1) signifying waiting at an intersection using a cell phone. The variables that link the two graphs are queue length, delay, and average traffic density at intersections. Queue length, delay, and average density are functions of the physical position of the vehicle (intersection characteristics, traffic light duration, and any random event) and the state of the connectivity to the communication network or the Internet. It is assumed that connection to the communication network is random. The behavior of the two networks together can be studied using the 
method of reflection triangles. Each side of the reflection triangle represents one of the (3) variables. One side would be the queue length, one side delay and the third side the average density. The angles of the reflection triangle represent the degree of the dependency of the variables to each other. For example, an acute angle between queue length and delay indicates that delay is much greater than queue length. On the other hand, if the angle between queue length and delay is large, then it indicates that the two variables correlate. An extreme case is when the angle between the two variables is (180 degrees). This implies that the two variables have the same magnitude. To test the methodology of reflection triangles, the evolution of an urban intersection is analyzed given the existence of a communication network that is connected to the intersection. By this, it is meant that all vehicles at the intersection are communicating via cell phones or Internet. The simulation method used for the analysis is the method of (Reflective Triangles (RF)) [3]-[5]. The complex Vehicular-Road network with reflective triangles is called a Reflective Network (RN).

Vehicular Networks $(\mathrm{VN})$ is a focus of study for many researchers. This is due to the evolution of wireless communication that allows for vehicle to vehicle, and vehicle to Internet communication. The advantages of the VN network are the use of VN in accident avoidance, traffic jam, and traffic delay regulation, routing modification, almost instant emergency aid access, and immediate collection and distribution of safety information. The bulk of the research on the VN is on designing ways to render it more efficiently [6] [7]. There is an abundant literature on rendering road networks efficiently and risk free effectively by reducing the impact on road users of network failure due to incident-related congestion and bottlenecks in particular, congestion from accidents, vehicle breakdowns, road works, lane blockages and road closures, [8] [9]. Up to now, there have been very few studies done on the impact of vehicular networks on road networks [10] [11]. The current main areas of research in this area are: in routing algorithms [12] [13] (e.g. shortest path algorithms), and adaptive traffic lights [14] [15]. There is no overlap between the research on the (VNs), and the research on road networks. This paper adds to the existing literature by introducing a new algorithm that is aimed at improving and facilitating road traffic by introducing a VN and thus constructing a complex multilayer network (Vehicle-Road network) and providing a means of analyzing such a complex system using the reflective triangles.

\section{The Building Blocks of the Vehicle-Road Network}

Each Reflective Network, RN is made of two or more networks. These networks are not necessarily compatible but can be compared through transformation into graphs. The graphs are related to each other by hyper-links. The representations of graphs are of outmost importance. For example, the urban road network should be represented as a stochastic Hamiltonian graph, and the Vehicle Network should be represented as a random graph. The stochastic Hamiltonian graph is defined as a graph $\left(H\left(X_{T}, E_{T}\right)\right)$ consisting of an ordered pair of disjoint sets $\left(X_{T}, E_{T}\right)$ such that for $T=\left(\left(t_{1}, \cdots, t_{k}\right) \in T\right)$ where each $\left(t_{i} ; i=1, \cdots, k\right)$ is a time point, $(T)$ is the study period, and the set $\left(X_{T}\right)$ is the set of all vertices during the study period $(T)$ such that $\left(X_{T}=\left(x_{t_{1}}, x_{t_{2}}, \cdots, x_{t_{k}}\right)\right)$. Each $\left(x_{t_{i}}=\left(x_{1}\left(t_{i}\right), x_{2}\left(t_{i}\right), \cdots, x_{n^{\prime}}\left(t_{i}\right)\right) ; n^{\prime}=1, \cdots, N^{\prime}\right)$ is a set of vertices at any time point $\left(t_{i}\right)$, and $\left(N^{\prime}\right)$ is the total number of vertices during each time point. Each vehicle traverses the set of vertices $\left(x_{t_{i}}\right)$ once and only once during each time point $\left(t_{i}\right)$. The set $\left(E_{T}\right)$ is the set of all edges during time $(T)$, where $\left(E_{T}=\left(e_{t_{1}}, e_{t_{2}}, \cdots, e_{t_{k}}\right)\right)$ in the urban network. Each

$\left(e_{t_{i}}=\left(e_{1}\left(t_{i}\right), e_{2}\left(t_{i}\right), \cdots, e_{m^{\prime}}\left(t_{i}\right)\right) ; m^{\prime}=1, \cdots, M^{\prime}\right)$ is a set of edges at any time point $\left(t_{i}\right)$, and $\left(M^{\prime}\right)$ is the total number of edges during each time point. The stochastic Hamiltonian graph includes cycles.

The Vehicle Network (VN) should be represented as a random graph. The reason for this choice is that it gives the possibility of attaching probabilities to the subsets of the set of random graph space of the VN. The VN is represented by a random graph $G(V(\rho), p)$, where $(G)$ is the probability space, and

$$
\left(p=p\left(E_{G}\right), 0 \leq p \leq 1\right)
$$

is the probability that edge $\left(E_{G}\right)$ exists. The set of vertices $(V)$ is not fixed; it changes during each time point $\left(t_{i}\right)$. Since the number of vertices in the $\mathrm{VN}$ depends on the number of vehicles at intersections in the road network, thus the vertices are functions of densities, generically represented as $(V=V(\rho))$, where $(\rho)$ is the traffic density during time point $\left(t_{i}\right)$ on links with intersection vertices. Link density $(\rho)$ is defined as the number of vehicles per unit area of a link.

Normally, the probability space contains graphs that are structured on a fixed set of distinct vertices, for ex- 
ample, $(V=(n)=(1, \cdots, n))$. Contrary to this, the set of vertices $(V=V(\rho))$ is a variable set. The elements of each set vary with the number of vehicles at an intersection. For the initial period $\left(t_{1}\right)$, the space $G\left(V\left(\rho_{1}\right), p_{1}\right)$ is defined by letting $\left(V\left(\rho_{1}\right)=V\left(\rho_{1}, t_{1}\right)=1, \cdots, n\left(\rho_{1}, t_{1}\right)\right)$ and the probability of occurrence $\left(p_{1}=1\right)$. This could be considered as an initial boundary condition for the space $G\left(V\left(\rho_{1}\right), p_{1}\right)$. The justification for the assumption of $\left(p_{1}=1\right)$ is that period $\left(t_{1}\right)$ is the birth of the probability space $G\left(V\left(\rho_{1}\right), p_{1}\right)$, and therefore it has to be taken as a sure event otherwise the space is nonexistent.

Theorem 1. In the initial probability space, the number of edges $\left(0<E_{G}\right)$ and the number of vertices $\left(n\left(\rho_{1}, t_{1}\right) \geq 2\right)$ have to be at least (2).

Proof: Let $(V)$ be a set with $(n)$ fixed elements, $(V=(0,1, \cdots, n))$. Let $\left(G_{0}\right)$ be a subset of $(V)$ such that $\left(G_{0} \subset[V]\right)$. Let $\left(G_{0}=0\right)$ be an empty set. The probability of the elementary event that the subset $\left(G_{0}\right)$ exists is equal to $\left(P\left(G_{0}\right)=P^{m} \times q^{\left(\left(\begin{array}{l}N \\ 2\end{array}\right)^{-m}\right)}\right)$, where $(m)$ is the number of edges and is equal to zero $(m=0)$. The probability of $(m=0)$ is $\left(P\left(G_{0}\right)=1\right)$. The elementary subset $\left(G_{0}\right)$ is in the set $[V],\left(G_{0} \subset[V]\right)$. This is a contradiction since based on the Ramsey theorem [16], for every integer $(r \in N)$, there exists an $(n \in N)$ such that every connected set of order at least $(n)$ contains $\left(K^{\prime}\right)$ induced connected subsets. In this case, $(r=0)$, and $(n \geq 0)$, therefore the set $[V]$ should have at least one connected sub graph, which excludes the elementary subset $\left(G_{0}\right)$.

The implication of this theorem is that only those time points are selected that contain VN links. Thus there may be gaps between intervals, and so there is no continuity of time points. The most basic VN contains at least one link.

The number of edges for the graph $G\left(V\left(\rho_{1}\right), p_{1}\right)$ is $\left(N=\left(\begin{array}{c}n\left(\rho_{1}, t_{1}\right) \\ 2\end{array}\right)\right)$ and the number of sub graphs is $\left(2^{N}\right)$. During the subsequent intervals, i.e., $\left(\left(t_{2}, \cdots, t_{k}\right) \in T\right)$ if the number of edges of sub graphs is $(0 \leq M \leq N)$, the probability space is $G\left(\left(V\left(\rho_{i}\right), p_{i}\right) ; i=2, \cdots, k\right)$, where $V\left(\rho_{i}\right)=\left(1, \cdots, n\left(\rho_{i}, t_{i}\right)\right)$. The number of edges of sub graphs is $M=\left(n\left(\rho_{i}, t_{i}\right)\right)$. The probability of occurrence of $(M)$ number of edges is $\left(P_{M}=P^{M} \times(1-P)^{N-M}=P^{M} \times(q)^{N-M} ; q=1-P\right)$. The probability of the edge belonging to a sub graph is $G_{M}\left(\left(V\left(\rho_{i}\right), p_{i}\right) ; i=2, \ldots . ., k\right)$, is $\left(p_{i}=P^{M}=\frac{1}{\left(\begin{array}{l}N \\ M\end{array}\right)}\right)$.

The probability of the number of edges of sub graphs $\left(0 \leq M^{\prime} \geq N\right)$, where the number of edges exceed $(N)$, the initial graph edge number is calculated as follows: let $\left(M^{\prime}\right)$ be defined as $\left(M^{\prime}=(N-M)+\delta \times N^{\prime}\right)$ where $\left(N^{\prime}\right)$ is the maximum allowable edges in the probability space $G(V(\rho), p)$, and $(\delta)$ is a fraction between $[0,1]$. This could occur if and only if $\left(\rho=\rho_{\max }\right)$ and all vehicles in the network were communicating using wireless phones or internet connections, then $\left(N^{\prime}=N^{\prime}\left(\rho_{\max }\right)=n\left(\rho_{\max }, t\right)\right)$, here $(t)$ stands for the time interval corresponding to the event of $\left(\rho=\rho_{\max }\right)$, and the probability of occurrence of a sub graph with $\left(M^{\prime}\right)$ edges is $\left(P_{M^{\prime}}=P^{M} \times P^{M^{\prime}}\right) .\left(P^{M^{\prime}}\right)$ is the probability of occurrence of $\left(M^{\prime}\right)$ edges is defined as:

$$
\left(P^{M^{\prime}}=\frac{1}{\left(\begin{array}{c}
N^{\prime} \\
M^{\prime}
\end{array}\right)}\right) \text {. }
$$

Theorem 2. If $\left(0 \leq M^{\prime} \geq N\right)$, then $\left(P_{M^{\prime}}=P^{M} \times P^{M^{\prime}}\right)$.

Proof: Let $(G)$ be the probability space $\left(G=G\left(M, P_{M}\right)\right)$, where $(0 \leq M \leq N)$, and let $\left(G^{\prime}\right)$ be the probability space $\left(G^{\prime}=G^{\prime}\left(M^{\prime}, P_{M^{\prime}}\right)\right)$, where $\left(M^{\prime}=(N-M)+\delta \times N^{\prime}\right)$. Both $(G)$ and $\left(G^{\prime}\right)$ are subsets of $G(V(\rho), p),\left(G=G\left(M, P_{M}\right) \in G(V(\rho), p)\right)$ and $\left(G^{\prime}=G^{\prime}\left(M^{\prime}, P_{M^{\prime}}\right) \in G(V(\rho), p)\right)$. Since the two events $(G)$, and $\left(G^{\prime}\right)$ are independent, and occur with probabilities $\left(P_{M}\right)$ and $\left(P_{M^{\prime}}\right)$, then let $(P)$ be the product 
measure of the two measures $\left(P_{M}\right)$ and $\left(P_{M^{\prime}}\right)$. This implies $\left(P=P_{M} \times P_{M^{\prime}}\right)$. Similarly $\left(P_{M^{\prime}}=P^{M} \times P^{M^{\prime}}\right)$.

The set of Hyper links per time point $\left(t_{i}\right)$ is designated by $L\left(h_{t_{i}}\right) .\left(h_{t_{i}}\right) \mathrm{s}$ are the nodes corresponding to hyper links, where the set

$$
\left(h_{t_{i}}=\left(h_{1}\left(t_{i}\right), h_{2}\left(t_{i}\right), \cdots, h_{l}\left(t_{i}\right)\right)\right)
$$

where (l) is the number of intersections in the road network. Each node $\left(h_{j}\left(t_{i}\right)\right)$, where $(j=1,2, \cdots l)$ has two sets of coordinates $\left(x_{t_{i}}^{*}, V\left(\rho_{i}, t_{i}\right)\right)$, where $\left(x_{t_{i}}^{*}\right)$ are the set of intersection nodes in the Hamiltonian network, $\left(H\left(X_{T}, E_{T}\right)\right)$, and $\left(V\left(\rho_{i}, t_{i}\right)\right)$ are the set of nodes in the vehicle network, $G(V(\rho), p)$ that are around intersection nodes. Since a hyper link represents the existence of wireless communication at intersections, it does not matter which link in the set of VN network is used as long as they are correlated with the right intersection nodes in the road network. Hyper links flag out the existence of wireless communication and their role does not extend beyond this. Each hyperlink is characterized by $(0,1),(0)$ signifying waiting at an intersection without using a cell phone, (1) signifying waiting at an intersection using a cell phone. The nodes $\left(h_{j}\right)$ are used to locate the parent triangles in reflective networks. In Figure 1, the communication links $(3,2)$, and $(1,2)$ represent internet connection or conversation with someone outside of the zone of the intersection by road users (1), and (3). Communication link $(1,3)$ represents that the two road users on links (1), and (3) are in communication with each other.

\section{The Method of Reflective Networks}

In this section a new algorithm is introduced that analyses and calculates the impact of a Vehicle Network on an urban traffic network. The methodology is based on the application of reflective triangles. Reflective triangles demonstrate the state of a network at intersections, and they reflect the state of the network in the near future. For example, if a period $(T)$ is chosen to study the network, and if this period is divided into $(n)$ equal intervals $(\sigma t)$, such as $\left(t=t_{1}, t_{2}, \cdots, T\right)$, then parent triangles are constructed during period $\left(t_{1}\right)$. During each subsequent interval offspring triangles are constructed based on the parent triangles. Each parent triangle can have an off spring or many off springs (siblings) during each period. The occurrence of multiple parents or multiple siblings depends on whether changes during an interval are static or micro-stochastic. Micro-stochastic refers to changes in variables that occur during an instance of time which from here on will be designated as $\left(\varepsilon_{\wedge}\right)$-changes. $\left(\varepsilon_{\wedge}\right)$ changes can be regarded as spikes in the values of variables used in constructing either parent or offspring triangles. Normally, the values of the variables used are continuous during each time point $\left(t_{i}\right)$, but any sudden changes due to unexpected events during a time point $\left(t_{i}\right)$ cause spikes, and thus allow for either multiple parents or multiple off springs. The details are given later on in this section.

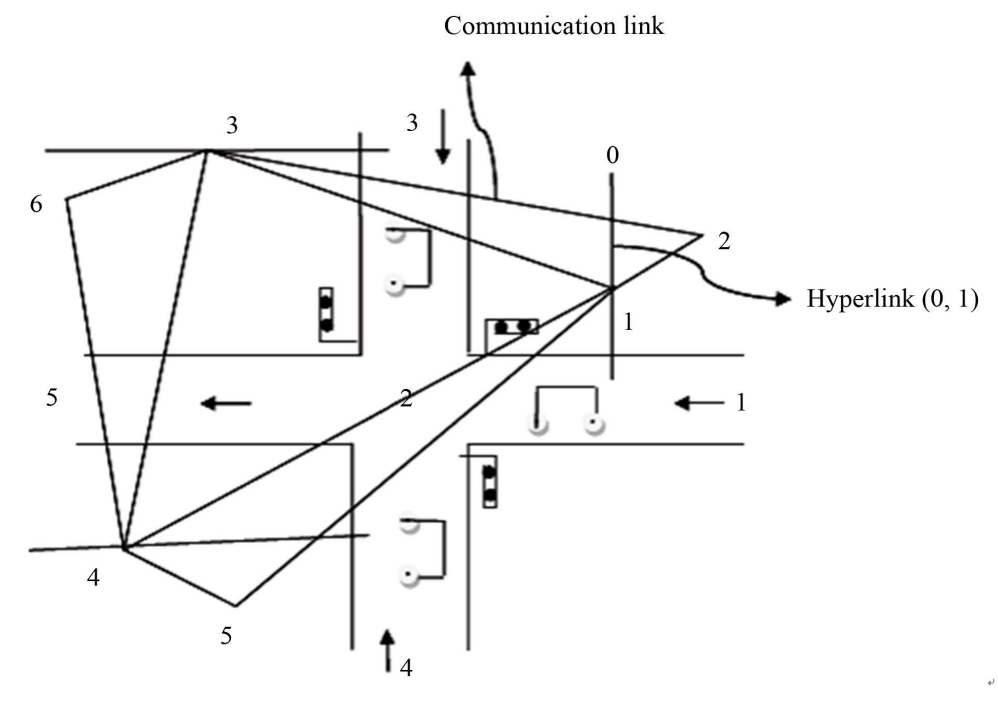

Figure 1. The Road-Vehicle Network with hyper links. 
The objective behind building reflective triangles is to define the dynamic nature of each intersection in a concrete manner, where a definite structure can be formed in a manner that is called "deterministic chaos". The evolution of initial or parent triangles to their off springs show the way to deciphering the ordered or chaotic nature of each dynamic system, which in this case is a Road-Vehicle Network represented by the characteristics and the communication activities around intersections. The second advantage of applying the method of reflective triangles is that it allows for an in depth analysis of a system without having to depend on historical data. Each time a system analysis is required, a whole new base data (parent triangles) could be constructed that reflect more realistically the nature of the system given that the environmental (external) and the internal vectors of the system are entirely different from the their historical counterparts.

The method of reflective triangles consists of building the parent triangle during the initial or starting period, and then constructing off-spring triangles for the consecutive intervals. The reflective or off spring triangle is obtained by reflecting the vertices of the parent triangle. The parent triangle is either generative or degenerative. A degenerative parent triangle is most likely to produce degenerative off springs which is an indicator of a chaotic evolution. Generative parent triangles are more likely to produce generative off springs. Obviously, each triangle is made up of three angles and three sides. The sum of the three angles must add up to 180 degrees; these angles are represented by $(\alpha),(\beta)$, and $(\gamma=180-(\alpha+\beta))$. Angles $(\alpha)$, and $(\beta)$ must be determined in a systematic manner each time a triangle is constructed. Each side of a triangle is representative of traffic and communication characteristics at an intersection. Intersection characteristics are designated as: queue length $(\vartheta)$, delay $(\Lambda)$, and traffic density $(\rho)$. Queue length, delay, and density are normally defined as functions of traffic behavior only. In the new formulation, these variables are formulated as functions of the physical position of a vehicle (intersection characteristics, and any random event) and the state of the connectivity to the communication network. The length of one side of a triangle represents queue length $(\vartheta)$ at intersection, the length of the second side represents traffic density, $(\rho)$, and the length of the third side represents delay $(\Lambda)$. The magnitude of the angles $(\alpha)$, and $(\beta)$ and $(\gamma)$ indicate the degree of the dependability of the three traffic characteristics at any intersection. The parent triangle is constructed during the initial time point $\left(t_{0}\right)$. The off spring triangle is born from the parent triangle during the next time point. Each off spring gives birth to its own off spring during the consequent time point. This way the parent triangle becomes the ancestor of the future generation off spring triangles.

The evolution from the parent triangle to the off spring (reflective) triangle and the next generations is depicted in Figure 2. In Figure 2, the intersection of Figure 1 is revisited. The objective is to demonstrate the evolution of traffic through reflective triangles. Initially, the parent triangle is a generative triangle, which indicates long queue

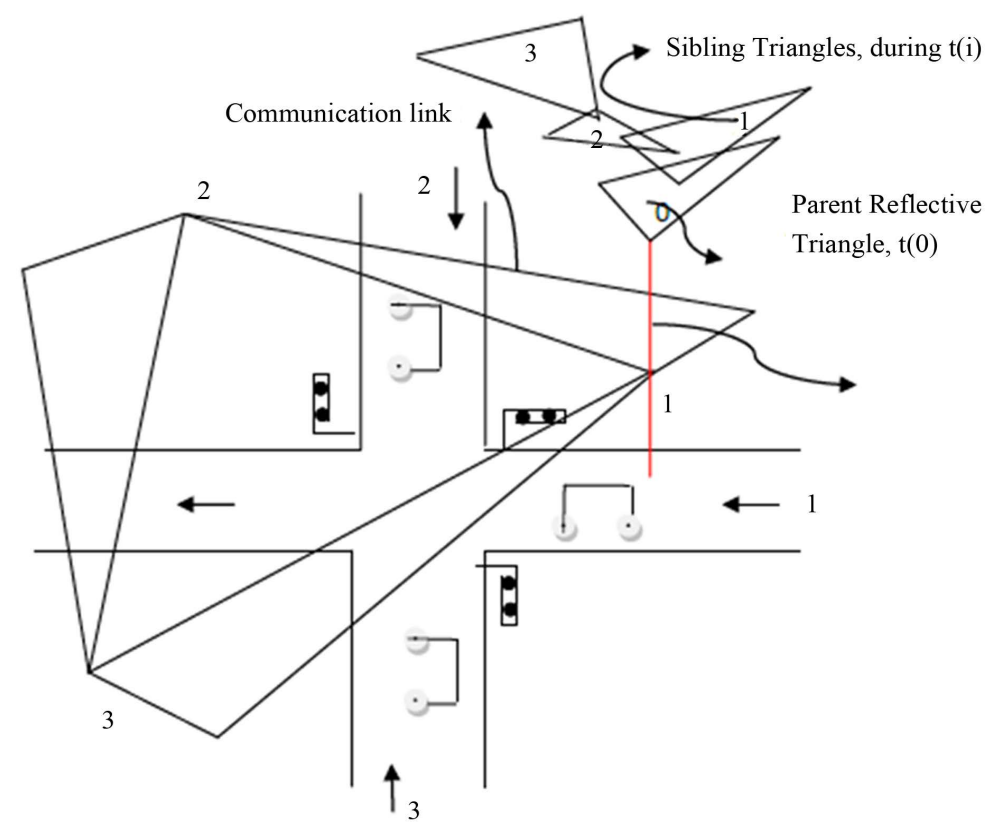

Figure 2. Parent triangle at $\left(t_{0}\right)$ and its off spring and the next generation at $\left(t_{i}\right) \mathrm{s}$. 
length $(\vartheta)$ and delay $(\Lambda)$ compared to density $(\rho)$. The acute angle between the queue length and delay confirms the correlation between the two variables. This situation is due to the existence of an active Vehicle Network. The off spring (reflective) triangle is generative, the queue length, delay, and density are similar in magnitude to the parent triangle and thus predict that if the Vehicle Network has a fixed number of edges, the traffic around the intersection will exhibit stable behavior and this will continue for many consecutive intervals.

The parent queue length $\left(\vartheta_{\left(P, t_{0}\right)}\right)$ is defined as the number of cars that are likely to remain at the intersection by the end of the initial time point. The parent queue length $\left(\vartheta_{\left(P, t_{0}\right)}\right)$ is calculated as a function of the number of cars entering the intersection during $\left(t_{0}\right),\left(d_{\left(\lambda, t_{0}\right)}\right)$, and the number of cars that are left on intersection links from before the initial time point, $\left(\eta_{\lambda}\right)$, and $\left(P_{M}\right)$ is the probability that $\mathrm{VN}$ has $(M)$ edges, as in Equation (1):

$$
\vartheta_{\left(P, t_{0}\right)}=\left(\sum_{\lambda} d_{\left(\lambda, t_{0}\right)}+\sum_{\lambda} \eta_{\lambda}\right)-\left(\sum_{\lambda} d_{\left(\lambda, t_{0}\right)}+\sum_{\lambda} \eta_{\lambda}\right)^{P_{M}}
$$

It is possible to have many parent or initial triangles during interval $\left(t_{0}\right)$ if there are micro-stochastic changes. Multiple parent triangles are created by generating several queue lengths as is given in Equation (2):

$$
\vartheta_{\left(e_{\wedge}, t_{0}\right)}=a_{\left(1, t_{0}\right)} \times \vartheta_{\left(P, t_{0}\right)}+\varepsilon\left(e_{\wedge}, t_{0}\right)
$$

In Equation (2), $(a)$ is a constant, and $(\varepsilon)$ is a small perturbation that causes a spike in the parent queue length $\left(\vartheta_{\left(P, t_{0}\right)}\right)$. The parent delay $\left(\Lambda_{\left(P, t_{0}\right)}\right)$ is defined as the number of cars that cross an intersection during the green time. This is expressed in Equation (3) as:

$$
\Lambda_{\left(P, t_{0}\right)}=\left(\sum_{\lambda} \delta_{\left(\lambda, t_{0}\right)} \times \mu\right)^{P_{M} \times n\left(\rho, t_{0}\right) \times c_{\left(l, t_{0}\right)}}
$$

In Equation (3), $\left(\delta_{\left(\lambda, t_{0}\right)}\right)$ represents the number of cars at an intersection ready to cross. $(\mu)$ is the duration of green light in seconds. $\left(n\left(\rho, t_{0}\right)=M\right)$ is the number of active communication edges during the time point $\left(t_{0}\right)$. $c_{\left(l, t_{0}\right)}$ is the average communication length in seconds during time point $\left(t_{0}\right)$. Similarly, to Equation (2), several micro-stochastic delays can be generated as in Equation (4):

$$
\Lambda_{\left(e_{\wedge}, t_{0}\right)}=a_{\left(2, t_{0}\right)} \times \Lambda_{\left(P, t_{0}\right)}+\varepsilon\left(e_{\wedge}, t_{0}\right)
$$

The average parent density $\left(\rho_{\left(P, t_{0}\right)}\right)$ is defined as average density for all links around an intersection during $\left(t_{0}\right),\left(\rho_{A v\left(P, t_{0}\right)}\right)$ is modified to include the impact of the $\mathrm{VN}$, as in Equation (5):

$$
\rho_{\left(P, t_{0}\right)}=\rho_{A v\left(P, t_{0}\right)}+\left(\rho_{A v\left(P, t_{0}\right)}\right)^{P_{M}}
$$

In Equation (5), the modified average parent density is added to the regular average density in order to reflect the impact of the communication network on links around an intersection. Many average parent densities can be generated through Equation (6):

$$
\rho_{\left(e_{\wedge}, t_{0}\right)}=a_{\left(3, t_{0}\right)} \times \rho_{\left(P, t_{0}\right)}+\varepsilon\left(e_{\wedge}, t_{0}\right)
$$

The angles $(\alpha),(\beta)$, and $(\gamma)$ are calculated as:

$$
\begin{gathered}
\alpha_{\left(P, t_{0}\right)}=\left(\frac{\Lambda_{\left(P, t_{0}\right)}}{\vartheta_{\left(P, t_{0}\right)}}\right) \times 180 \\
\beta_{\left(P, t_{0}\right)}=\left(\frac{\rho_{\left(P, t_{0}\right)}}{\vartheta_{\left(P, t_{0}\right)}}\right) \times 180 \\
\gamma_{\left(P, t_{0}\right)}=180-\left(\alpha_{\left(P, t_{0}\right)}+\beta_{\left(P, t_{0}\right)}\right)
\end{gathered}
$$


Similarly, the angles corresponding to several parent triangles can be calculated. The parent triangle is constructed using a unit disk $(D=r \in C ;|r|=1)$, where $(r)$ is the radius [16]. As a subset of disk $(D)$, a circle is constructed with radius $(R)$ equal to $(r)$ times the parent queue length $\left(R=r \times \vartheta_{\left(P, t_{0}\right)}\right)$. The center of the circle is the coordinates of the hyperlink at an intersection. From a point on the periphery of the circle, a line the size of $\left(\Lambda_{\left(P, t_{0}\right)}\right)$ is drawn at an angle $(\alpha)$ from the radius. The radius can be drawn at any angle. A line the size of $\left(\rho_{\left(P, t_{0}\right)}\right)$ at an angle $(\beta)$ with the line $\left(\Lambda_{\left(P, t_{0}\right)}\right)$ is drawn that intersects the radius $(R)$. The radius and the density line intersect at an angle $(\gamma)$. This is demonstrated in Figure 3.

If micro-stochastic events occur, then other parent triangles can be constructed given Equations (2), (4) and (6). The reflection or off spring triangle can be constructed from the parent triangle through the reflection of the vertices of the parent triangle. This is obtained by applying the formula suggested by [5]. The first step in constructing the off spring triangle is to design an operator that can reflect the vertices of the parent triangle. Let the operator be denoted by $T=\left[s s^{\prime}, p p^{\prime}\right]$. The two components of the operator are obtained as follow:

$$
\begin{gathered}
s=\sin ^{2}\left(\alpha_{\left(P, t_{0}\right)}\right)+\sin ^{2}\left(\beta_{\left(P, t_{0}\right)}\right)+\sin ^{2}\left(\gamma_{\left(P, t_{0}\right)}\right), \\
p=\sin ^{2}\left(\alpha_{\left(P, t_{0}\right)}\right) \times \sin ^{2}\left(\beta_{\left(P, t_{0}\right)}\right) \times \sin ^{2}\left(\gamma_{\left(P, t_{0}\right)}\right) . \\
s s^{\prime}=\frac{\left[(s+16 p) \times(4 s-5)^{2}\right]}{[4 s+1+64 p \times(4 s-7)]} \\
p p^{\prime}=\frac{\left[p \times(4 s-5)^{6}\right]}{[4 s+1+64 p \times(4 s-7)]^{2}} .
\end{gathered}
$$

The coefficients in Equation (11), are the ones suggested by IJzeren [5], but in general any coefficient would work as long as $\left(\left(s s^{\prime}, p p^{\prime}\right) \neq\left(\frac{1}{2}, \frac{1}{2}\right)\right)$, and $\left(s s^{\prime} \times p p^{\prime} \neq 1\right)$. This condition is demonstrated by [16]. The same condition also assures the non-degeneracy of the off spring triangles. The first step towards obtaining an off spring or a reflective triangle, is to reflect the vertices of the parent or (initial) triangle. To find the reflective vertices, a reflection circle of radius $\left(r_{1}\right)$, the size $\left(r_{1}=\frac{\vartheta_{\left(P, t_{0}\right)}}{s s^{\prime}}\right)$ is drawn. The reflection circle crosses the parent queue length $\left(\vartheta_{\left(P, t_{0}\right)}\right)$ at point $\left(a_{1}\right)$. The second reflection circle of radius $\left(r_{2}\right)$, the size $\left(r_{2}=\frac{\Lambda_{\left(P, t_{0}\right)}}{p p^{\prime}}\right)$ is drawn. The second reflection circle crosses the parent delay $\left(\Lambda_{\left(P, t_{0}\right)}\right)$ at point $\left(b_{1}\right)$. Both reflection circles cross the parent average density at points $\left(c_{1}^{\prime}\right)$ and $\left(c_{2}^{\prime}\right)$ as is shown in Figure 4 . The three points $\left(a_{1}\right),\left(b_{1}\right)$, and $\left(c_{1}=\frac{c_{1}^{\prime}+c_{2}^{\prime}}{2}\right)$ are the reflections of the (3) vertices of the parent triangle. It is postulated that the three points $\left(a_{1}\right),\left(b_{1}\right)$, and $\left(c_{1}\right)$ are in fact the points of intersections of the bisectors of the parent triangle with its sides. The point of intersection of the bisectors of the parent triangle $(O)$ is the starting point of the off spring or reflection triangle.

The off spring or reflective queue length $\left(\vartheta_{\left(R, t_{i}\right)}, i \neq 0\right)$ is calculated by Equation (12) as follows:

$$
\vartheta_{\left(R, t_{i}\right)}=\left(\frac{\vartheta_{\left(P, t_{0}\right)}}{s s^{\prime}}+\sum_{\lambda} d_{\left(\lambda, t_{i}\right)}\right)-\left(\frac{\vartheta_{\left(P, t_{0}\right)}}{s s^{\prime}}+\sum_{\lambda} d_{\left(\lambda, t_{i}\right)}\right)^{P_{M^{\prime}}}
$$

$\left(P_{M^{\prime}}\right)$ is the probability that there are $\left(M^{\prime}\right)$ communication links during. The off spring or reflective delay $\left(\Lambda_{\left(R, t_{i}\right)}, i \neq 0\right)$ is calculated by Equation (13) as follows: 


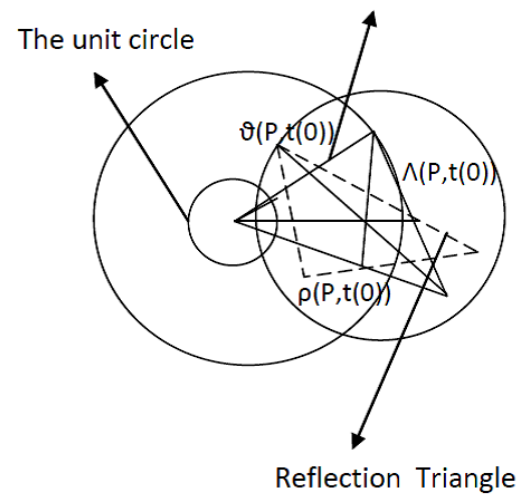

Figure 3. Parent and reflection triangles drawn from subsets of disk (D).

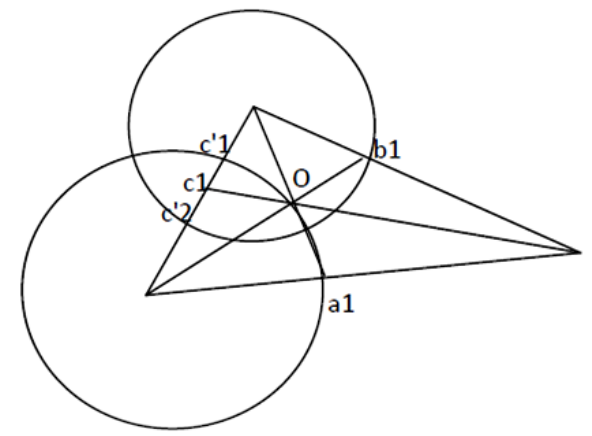

Figure 4. Method of bisectors.

$$
\Lambda_{\left(R, t_{i}\right)}=\frac{\Lambda_{\left(P, t_{0}\right)}}{p p^{\prime}}+\left(\sum_{\lambda} \delta_{\left(\lambda, t_{i}\right)} \times \mu\right)^{P_{M^{\prime} \times n}\left(\rho, t_{i}\right) \times c_{\left(l, t_{i}\right)}}
$$

The green time $(\mu)$ is fixed for all time points. $\left(n\left(\rho, t_{i}\right)=M^{\prime}\right)$ is the number of active communication edges during the time point $\left(t_{i}\right) \cdot c_{\left(l, t_{i}\right)}$ is the average communication length during $\left(t_{i}\right)$. The average off spring or reflective density is calculated as in Equation (14):

$$
\rho_{\left(R, t_{i}\right)}=\rho_{A v\left(R, t_{i}\right)}+\left(\rho_{A v\left(R, t_{i}\right)}\right)^{P_{M^{\prime}}}
$$

$\left(\rho_{\left(R, t_{i}\right)}\right)$ is the average of the densities of all the links of an intersection during $\left(t_{i}\right)$. The angles corresponding to the (3) sides of the off spring triangle are calculated as follow:

$$
\alpha_{\left(R, t_{i}\right)}=\left(\frac{\Lambda_{\left(R, t_{i}\right)}}{\vartheta_{\left(R, t_{i}\right)}}\right) \times 180
$$

and

$$
\begin{gathered}
\beta_{\left(R, t_{i}\right)}=\left(\frac{\rho_{\left(R, t_{i}\right)}}{\vartheta_{\left(R, t_{i}\right)}}\right) \times 180 \\
\gamma_{\left(R, t_{i}\right)}=180-\left(\alpha_{\left(R, t_{i}\right)}+\beta_{\left(R, t_{i}\right)}\right)
\end{gathered}
$$

It is possible to have more than one off spring triangle; in other words an off spring can have siblings. This would be the result of the occurrence of micro-stochastic events during $\left(t_{i}\right)$. In that case, the off shoots of the three sides of the off spring triangle are expressed as follow: 


$$
\begin{aligned}
& \vartheta_{\left(e_{\wedge}, t_{i}\right)}=a_{\left(1, t_{i}\right)} \times \vartheta_{\left(R, t_{i}\right)}+\varepsilon\left(e_{\wedge}, t_{i}\right) \\
& \Lambda_{\left(e_{\wedge}, t_{i}\right)}=a_{\left(2, t_{i}\right)} \times \Lambda_{\left(R, t_{i}\right)}+\varepsilon\left(e_{\wedge}, t_{i}\right) \\
& \rho_{\left(e_{\wedge}, t_{i}\right)}=a_{\left(3, t_{i}\right)} \times \rho_{\left(R, t_{i}\right)}+\varepsilon\left(e_{\wedge}, t_{i}\right)
\end{aligned}
$$

Calculation of the corresponding angles for the sibling triangles is similar to the off spring triangle. It suffices to replace the variables by their micro-stochastic versions. The off spring triangle is constructed following the procedure described here. As is shown in Figure 3, to construct the reflection or off spring triangle, one must draw the angle bisectors of the parent triangle, details of which are given earlier. The point of the intersection of the three angles bisectors becomes the center of a new reflection circle, subset of the disk $(D)$. The radius of the reflection circle is the size of the off spring queue length $\left(\vartheta_{\left(R, t_{i}\right)}\right)$. From the center of the reflection circle, one of the angle bisector lines is extended to the size of the off spring queue length $\left(\vartheta_{\left(R, t_{i}\right)}\right)$ in order to get a point on the periphery of the reflection circle as is shown in Figure 3. From the point on the periphery, a line the size of the off spring delay $\left(\Lambda_{\left(R, t_{i}\right)}\right)$ at an angle $\left(\alpha_{\left(R, t_{i}\right)}\right)$ is drawn. The second line the size of the average off spring density $\left(\rho_{\left(R, t_{i}\right)}\right)$ at an angle $\left(\beta_{\left(R, t_{i}\right)}\right)$ with $\left(\Lambda_{\left(R, t_{i}\right)}\right)$ is drawn. The angle formed by the intersection of the queue length $\left(\vartheta_{\left(R, t_{i}\right)}\right)$ and the average off spring density $\left(\rho_{\left(R, t_{i}\right)}\right)$ is automatically equal to $\left(\gamma_{\left(R, t_{i}\right)}\right)$.

\section{Case Study}

In order to show the validity of the methodology introduced in this paper, an intersection during two time periods, $\left(t_{1}\right)$ and $\left(t_{2}\right)$ is chosen. An interval is the time gap between two green times. It is assumed that during $\left(t_{1}\right)$, some residue of traffic from the previous period is still at the intersection waiting to exit. The residual queue is $\left(\sum_{\lambda} \eta_{\lambda}=\left[\begin{array}{lll}1 & 1 & 1\end{array}\right]\right)$, meaning that the intersection has (3) links and each link has (1) car still waiting to exit. The number of cars entering each link of the intersection during the initial period is $\left(\sum_{\lambda} d_{\left(\lambda, t_{1}\right)}=\left[\begin{array}{lll}1 & 1 & 1\end{array}\right]\right)$. It is assumed that all the cars stored at the intersection use cell phones, and thus the number of links of the VN is equal to the number of cars. The probability of the existence of such a Vehicle Network is calculated. The Parent queue length is calculated according to Equation (1). The parent queue length constitutes one side of the parent or initial triangle. The parent delay and the average parent density and the three angles are calculated using Equations (3), (5) and (7)-(9). In this case, no micro stochastic events are introduced. Therefore, there is one parent triangle during $\left(t_{1}\right)$, and one off spring triangle during $\left(t_{2}\right)$. The parent queue length is calculated to be equal to (4.9062765 cars). The parent delay is ( 1.933182 cars), and the average parent density is ( 2 cars). The magnitude of the angles correspond to the magnitude of the sides. Angle $\left(\alpha_{\left(P, t_{1}\right)}\right)$ is equal to (70.924003 degrees), angle $\left(\beta_{\left(P, t_{1}\right)}\right)$ is equal to (73.375401 degrees), and angle $\left(\gamma_{\left(P, t_{1}\right)}\right)$ is equal to (35.700596 degrees). The parent triangle for the intersection is presented in Figure 5.

The data requirements for the second interval are: the duration of green time $(\mu)$ is equal to ( 3 seconds). The average communication length $\left(c_{\left(l, t_{1}\right)}\right)$ is equal to $(60$ seconds). The number of cars entering each link of the intersection during the second period is $\left(\sum_{\lambda} d_{\left(\lambda, t_{2}\right)}=\left[\begin{array}{lll}1 & 1 & 2\end{array}\right]\right)$. The off spring queue length is calculated using Equation (3.12) and is equal to (5.9230318 cars). The off spring delay is equal to (1.4383141 cars), and the average off spring density is equal to ( 2 cars). Angle $\left(\alpha_{\left(R, t_{2}\right)}\right)$ is equal to (43.726924 degrees), angle $\left(\beta_{\left(R, t_{2}\right)}\right)$ is equal to (60.803024 degrees), and angle $\left(\gamma_{\left(R, t_{2}\right)}\right)$ is equal to (75.470052 degrees). The off spring or reflection triangle for the intersection is presented in Figure 6.

Figure 7 presents the parent and the off spring triangles together. 


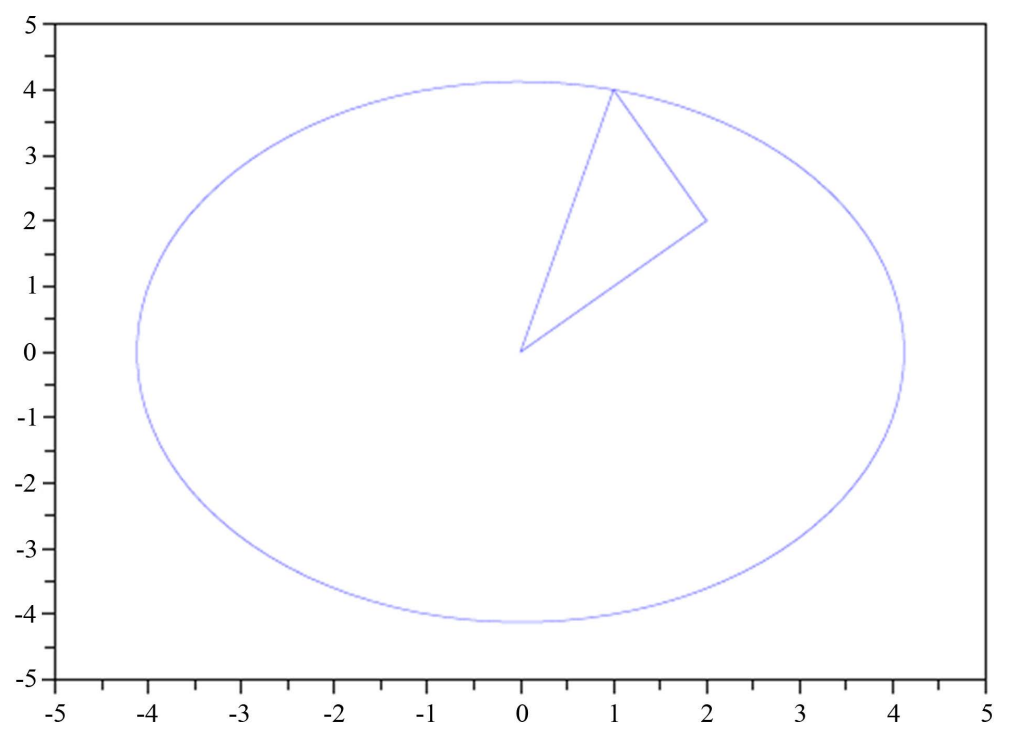

Figure 5. The parent triangle.

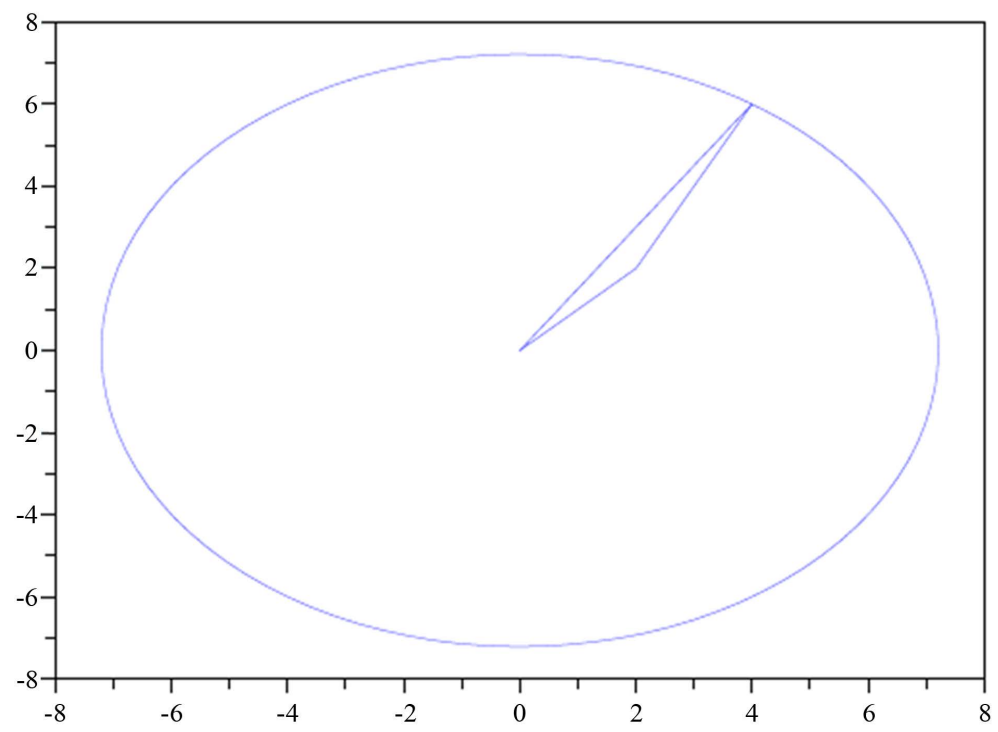

Figure 6. The off spring or reflection triangle.

\section{Conclusion}

Reflective network is an urban network connected to a vehicle network through hyperlinks. It is called reflective network because it contains reflective triangles. In this type of an urban network, each intersection is represented by a multitude of triangles, each one representing the state of traffic at an intersection during a time interval. Each triangle is constructed based on the previous triangle given a particular procedure. The process is repeated and each triangle is analyzed after each iteration. The set of triangles forms a dynamic system which could demonstrate either a stable (generative triangles) or a chaotic (degenerative) evolution depending on the situation of traffic and the state of communication at intersections. The advantage of such a method is primarily that it allows for a complex multilayer network that consists both of physical links (roads) and communication links that can be related to each other. The dependency on historical data is significantly reduced since for each situation, it is now possible to create its own particular history starting with the parent triangle to the off spring triangle and the next generations. The ancestor triangle constitutes the historical data. The method of reflection triangles also allows for 


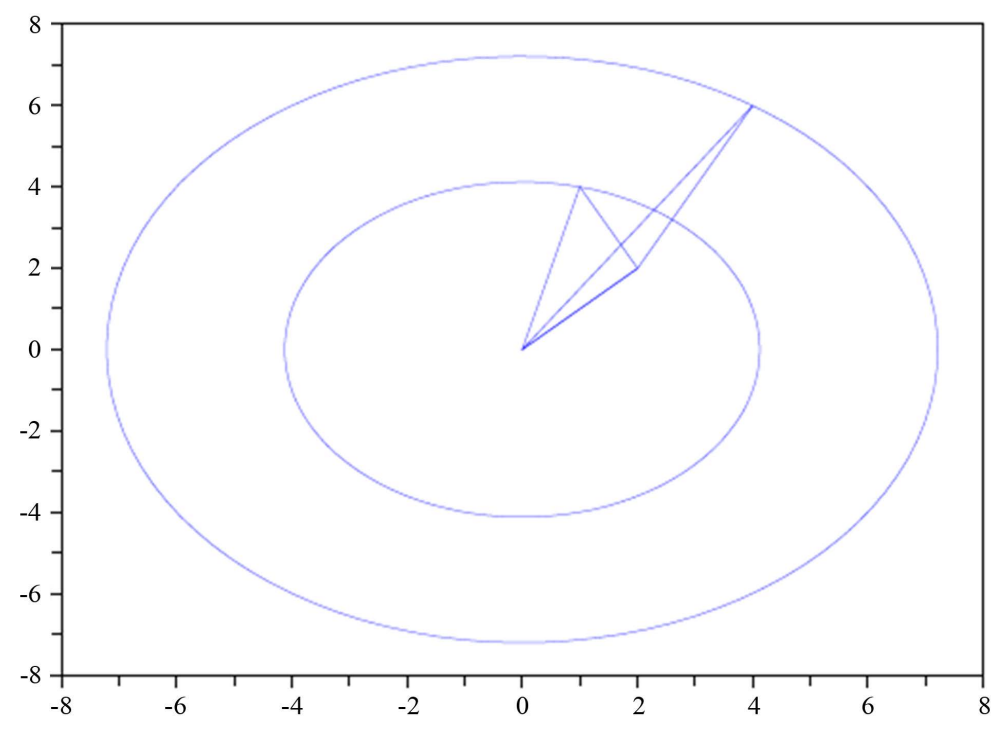

Figure 7. The parent and the off spring triangles.

a rapid macroscopic analysis of the system through the parent and the reflection triangles of all intersections included in the reflective network. The method of reflective networks opens a whole new way of analyzing traffic by focusing at intersections rather than tracking traffic along each link of a network.

\section{References}

[1] Bollobâs, B. and Erdos, P. (1976) Cliques in Random Graphs. Mathematical Proceedings of the Cambridge Philosophical Society, 80, 419-427. http://dx.doi.org/10.1017/S0305004100053056

[2] Bollobâs, B. (2000) Modern Graph Theory. Springer, Berlin.

[3] Nicollier, G. (2013) Convolution Filters for Triangles. Forum Geometricorum, 13, 61-85.

[4] Ismailescu, D. and Jacobs, J. (2006) On Sequences of Nested Triangles. Periodica Mathematica Hungarica, 53, 169184. http://dx.doi.org/10.1007/s10998-006-0030-3

[5] van IJzeren, J. (1984) Driehoeken met gegeven spiegelpuntsdriehoek. EUI Report, 84-WSK-03, 356-373.

[6] Cayford, R. and Johnson, T. (2003) Operational Parameters Affecting the Use of Anonymous Cell Phone Tracking for Generating Traffic Information. Transportation Research Board Annual Meeting, Technical Report, Washington DC.

[7] Hartenstein, H., Bochow, B., Ebner, A., Lott, M., Radimirisch, M. and Vollmer, D. (2001) Position-Aware Ad Hoc Wireless Networks for Inter-Vehicle Communications: The Fleetnet Project. Proceedings of the Second ACM International Symposium on Mobile and Ad Hoc Networking and Computing (MobiHoc01), Long Beach, 4-5 October 2001, 259-262.

[8] Gonzalez, M.C., et al. (2008) Understanding Individual Human Mobility Patterns. Nature, 453, 779-782. http://dx.doi.org/10.1038/nature06958

[9] Bando, M., Hasebe, K., Nakayama, A., Shibata, A. and Sugiyama, Y. (1995) Dynamical Model of Traffic Congestion and Numerical Simulation. Physical Review, 51, 1035-1042.

[10] Hsiao, W.C.M. and Chang, S.K.J. (2005) Segment Based Traffic Information Estimation Method Using Cellular Network Data. IEEE Intelligent Transportation Systems Conference, Vienna, 13-15 September 2005, 142-147.

[11] Gundlegard, D. and Karlsson, J. (2006) Generating Road Traffic Information from Cellular Networks-New Possibilities in UMTS. Proceedings of the 6th International Conference on ITS Telecommunications, Chengdu, 21-23 June 2006, 1128-1133.

[12] Lochert, C., Hartenstein, H., Tian, J., Füßler, H., Hermann, D. and Mauve, M. (2000) A Routing Strategy for Vehicular Ad Hoc Networks in City Environments. Project Report, within the Framework of the FleetNet Project as Part of BMBF Contract No. 01AK025D and Support from EU IST Project CarTalk (IST-2000-28185).

[13] Leontiadis, I. and Mascolo, C. (2007) GeOpps: Geographical Opportunistic Routing for Vehicular Networks. Project report EPSRC through Project Cream. IEEE International Symposium, Espoo, 24-28 June 2007, 1-6.

[14] Chaudhary, N.A., Kovvali, V.G. and Alam, S.M. (2002) Guidelines for Selecting Signal Timing Software. Product 
0-4020-P2. Texas Transportation Institute, College Station.

[15] Lobiya, N. and Lobiya, D.K. (2012) Performance Evaluation of Realistic Vanet Using Traffic Light Scenario. International Journal of Wireless and Mobile Networks (IJWMN), 4, 237.

[16] Nakamura, H. and Oguiso, K. (2003) Elementary Moduli Space of Triangles and Iterative Process. Journal of Mathematical Science, 10, 209-224. 
Scientific Research Publishing (SCIRP) is one of the largest Open Access journal publishers. It is currently publishing more than 200 open access, online, peer-reviewed journals covering a wide range of academic disciplines. SCIRP serves the worldwide academic communities and contributes to the progress and application of science with its publication.

Other selected journals from SCIRP are listed as below. Submit your manuscript to us via either submit@scirp.org or Online Submission Portal.
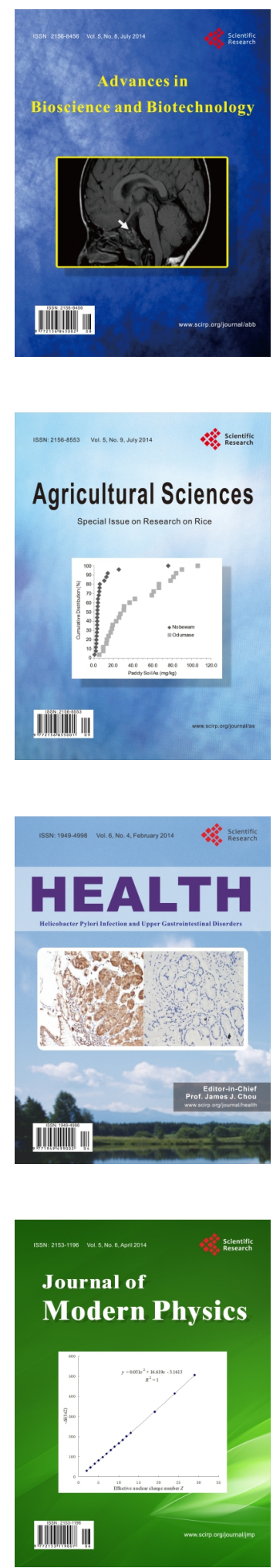
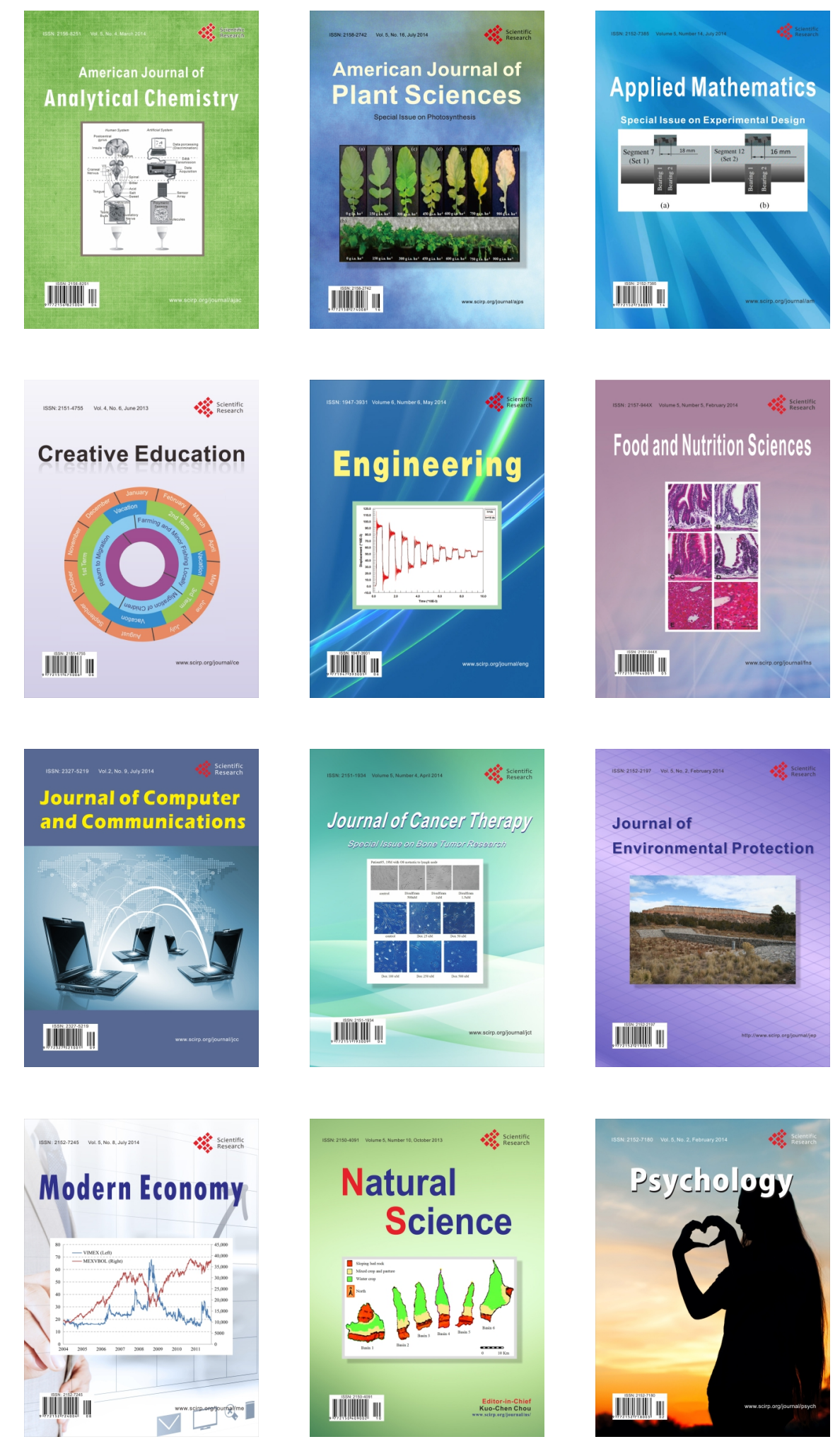